\title{
Review
}

\section{A Comprehensive Review on Surgical Implications of Rheumatoid Arthritis}

\author{
Olivia Foster, Nikolaj Brown, Nikhale Malik, Zaiba Akhtar, Mala Thakur* \\ Xavier University School of Medicine, Oranjestad, Aruba
}

${ }^{*}$ Correspondence to: Mala Thakur; Xavier University School of Medicine, Oranjestad, Aruba; E-mail: mala.thakur@students.xusom.com

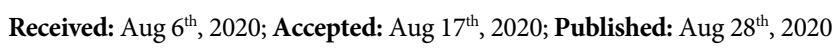

Citation: Foster O, Brown N, Malik N, Akhtar Z, Thakur M. A comprehensive review on surgical implications of rheumatoid arthritis. Gen Surg Open A Open J. 2020; I(1): 28-34.

\begin{abstract}
This paper focuses on Rheumatoid Arthritis (RA) in terms of its causative elements and the treatment options. Though there is yet to be a definite cause for this disease, this study breaks down its progression and the steps that lead to rheumatoid arthritis. The research presented here delves into the different joints, the areas affected, and the manifestations of the disease among patients. Towards the end, however, the paper discusses the triumphs made in medicine that are in the form of innovative drugs and treatments that treat and hinder the progression of this disease. This paper differentiates the various medication and sets out to clarify the advantages and disadvantages of the broadly classified rheumatoid arthritis medication. As it concludes, the paper also outlines the current surgical options available and offers alternatives and studies that enhance overall RA treatment.
\end{abstract}

Keywords: Arthrodesis, Arthritis, Orthopedics, Rheumatoid, Surgery, Synovectomy.

\section{INTRODUCTION \& BACKGROUND}

Rheumatoid Arthritis (RA) is an autoimmune, inflammatory disease that primarily affects joints and may be further complicated through certain extra-articular manifestations. In general, RA presents with symmetrical joint disease with synovitis. This illness chiefly affects the proximal interphalangeal and metacarpophalangeal joints, the wrist joints and may affect many others. Furthermore, RA may progress to extra-articular manifestations, which can occur in a variety of organs, including in the skin, lungs, heart, eyes, gastrointestinal system, renal system, and nervous system. ${ }^{1}$

Worldwide, RA affects approximately $1 \%$ of the total global population, and women are two to three more likely to be affected than men. ${ }^{2,3}$ Primarily, RA affects individuals within the 50-75 age range. To date, though it is well-established that RA is due to an autoimmune destructive process, research still has not identified the exact cause. It is widely hypothesized that the cause of RA is multifactorial. The incidence of RA is commonly associated with the presence of HLA DR4 and HLA DR1, and it is often triggered by environmental factors, including tobacco smoke and infection. ${ }^{4}$

Commonly accepted pathophysiology of RA is that environmental triggers lead to the citrullination of arginine residues in many proteins, including Type II collagen and Vimentin found in the joints. Antigen Presenting Cells (APCs) from genetically susceptible individuals (HLA-DR4 and HLA-DR1 positive) no longer recognize these as self-antigens, and they mount an immune response. Serum from RA patients may contain anti-citrullinated protein antibodies (ACPA) antibodies which bind to citrullinated proteins lead to complement activation. Anti-Rheumatoid factor positive RA patients are at greater risk of extra-articular manifestations. ${ }^{5}$

Furthermore, Th1, Th17, plasma, and B cells all secrete cytokines within the synovial space, including IL-1, IL- 6 , and TNF- $\alpha$, which induce inflammation as well as the attraction of other immune cells. The inflammatory cells lead to synovial cell proliferation and the formation of a pannus. The inflammatory cytokines also lead to the generation of osteoclasts, leading to bone erosion. ${ }^{6}$

A diagnosis of rheumatoid arthritis is largely based on clinical symptoms including morning stiffness and symmetrical arthritis at the wrist, hands, and feet. The diagnosis is typically made in conjunction with laboratory findings including the occurrence of anti-rheumatoid factor and anti-cyclic citrullinated peptide antibodies.

Currently, as there is no cure for RA, treatment has been divided into short-term and long-term. Short-term treatment for acute flare-ups has been largely aimed at symptom management. Non-Steroidal Anti-Inflammatory Drugs (NSAIDs) and glucocorticoids have sustained positive results in pain reduction and decreasing inflammation. However, these treatments do not affect the progression of the disease.7 Furthermore, glucocorticoids are typically not recommended for chronic use due to side effects including immunosuppression, weight 
gain, and bone thinning. ${ }^{8}$

Long-term treatment aims at delaying the progression of RA and minimizing subsequent joint destruction. Disease-Modifying Anti-Rheumatic Drugs (DMARDs) have shown effectiveness in decreasing and delaying disease progression. ${ }^{2}$ Traditional synthetic DMARDs include azathioprine, methotrexate, cyclosporine, and minocycline. Methotrexate is often considered the gold-standard treatment for many patients with RA. Biological DMARDs, including TNF-inhibitors (adalimumab, golimumab, certolizumab, and infliximab), anti-CD20 antibodies (rituximab), and anti-IL-6 antibodies (tocilizumab) are also commonly used in clinical practice. ${ }^{2}$ However, these drugs are usually used in conjunction with traditional DMARDs and are reserved for patients unresponsive to methotrexate. Early DMARD administration typically leads to better patient outcomes. However, despite the current therapies implemented, many patients do not achieve long-term remission; thus, more effective therapies are required.

In some patients, Arthroplasty may be indicated as a last resort in order to restore joint function or to relieve intractable pain in which medication has failed. It has been shown that RA patients between 40 and 59-years-old have decreased rates of arthroscopy as compared to those older than $60 .{ }^{9}$ Various surgeries that may be used in RA management include tenosynovectomy, tendon repair surgery, arthrodesis, and replacement arthroplasty. This article aims to assess the implications of surgical therapy on RA patients.

\section{REVIEW}

\section{Clinical Manifestations of Rheumatoid Arthritis}

Rheumatoid Arthritis RA presents with an array of clinical manifestations that are either systemic or articular. Systemic manifestations include general signs and symptoms such as fatigue, fever, rash, and weight loss. As with other autoimmune disorders, fatigue in RA patients may be severe because there is an increased risk for inflammation and chronic musculoskeletal disorders as joints involved can become deformed and disabled. Other systemic clinical manifestations that may be present in severe cases include interstitial lung disease, pleuritis, and pericarditis, among others. ${ }^{10,11}$ Articular manifestations are determined through the four signs of inflammatory response which are warmth, swelling, pain, and erythema. ${ }^{12}$

The onset of the RA articular symptoms is insidious and reported to be accumulative. They are classified as presenting for a duration longer than six weeks and are polyarticular and symmetrical. However, it lacks a definite protocol and early onset can present as asymmetrical. ${ }^{13}$ Articular morning stiffness, a common phenomenon amongst patients, lasts for over one hour and improves with activity throughout the day. ${ }^{12,14}$ Joint involvement tends to primarily involve the small peripheral joints: Metacarpophalangeal (MCP), Proximal Interphalangeal Joints (PIP), Intercarpal, and Carpometacarpal. Beyond the phalangeal joints, the cervical spine, acromioclavicular, and patellofemoral joints follow in incidence. ${ }^{13,15}$ The affected joints exhibit the cardinal signs of inflammation with pain from the hypertrophic synovial tissue classified as nociceptive pain. ${ }^{16}$

RA can take on a variety of presentations depending on the region of the affected joint. With the knee advanced RA can be identified with a Baker's cyst (synovial fluid collection behind the knee). In the phalanges of the upper extremities a Swan Neck manifestation can occur when there is distal interphalangeal (DIP) hyperflexion with proximal
PIP hyperextension. Boutonnière deformity, a PIP flexion with DIP hyperextension, and Z-thumb, the hyperextension of an interphalangeal joint, subluxation metacarpophalangeal joint, and fixed flexion of the carpometacarpal joint, can also manifest within the hands due to severe RA. Within the wrist ulnar deviation can occur which is when the fingers tend to drift towards the ulnar due to inflammatory damage to the ligaments and joints. In the phalanges of the foot a hammertoe can progress. This is when the proximal interphalangeal joint appears to be bent especially on the $2 \mathrm{nd}$, $3 \mathrm{rd}$, or 4 th toe due to the muscles and ligaments damage. Due to monitoring and early pharmacological intervention these deformities are no longer common. ${ }^{13,17}$

Articular signs and symptoms in RA patients should be quantified according to the 2010 Rheumatoid Arthritis Classification Criteria (Table 1.) on the area and pain level prompted on moving or palpating the joint. Twenty-eight joints are examined on tenderness and swelling; these are then quantified, recorded, and differentiated between true articular swelling and non- or periarticular involvement. ${ }^{18}$

Table 1. A summary of Surgical Intervention for Rheumatoid Arthritis

\begin{tabular}{|l|l|l|}
\hline Surgery type & Procedure involved & $\begin{array}{l}\text { Results observed after the } \\
\text { surgery }\end{array}$ \\
\hline Synovectomy & $\begin{array}{l}\text { The surgery entails the removal } \\
\text { of the inflamed synovium tissue }\end{array}$ & $\begin{array}{l}\text { The surgery helps manages pain } \\
\text { and reduces stiffness in the affected } \\
\text { part }\end{array}$ \\
\hline Arthrodesis & $\begin{array}{l}\text { The surgery is achieved by } \\
\text { removing the damaged joint } \\
\text { Fusing the bones }\end{array}$ & $\begin{array}{l}\text { the functioning of the joint the af- } \\
\text { fected part attains mobility }\end{array}$ \\
\hline Replacement & $\begin{array}{l}\text { Permanent or partial replace- } \\
\text { ment of the part of the joint }\end{array}$ & $\begin{array}{l}\text { The surgery helps the patient in } \\
\text { recovering the mobility of the af- } \\
\text { fected part. For example, movement } \\
\text { of the wrist }\end{array}$ \\
\hline
\end{tabular}

As an autoimmune disorder, the inflammatory response of RA can be measured and monitored through laboratory values. Most patients exhibit signs of hypoalbuminemia, thrombocytosis, high C-reactive proteins (CRP) and a high erythrocyte sedimentation rate (ESR). $80 \%$ of RA patients test positive for IgM rheumatoid factor (RF), autoantibodies against the Fc portion of IgG. ${ }^{19}$ Although RF is an important laboratory finding for RA, approximately $5 \%$ of a population always has a positive test for RF, while less than $0.4 \%$ of the population will actually have RA. When testing for Rheumatoid factor in the serum anti-CCP (cyclic citrullinated peptide) antibodies need to be measured as well. Together they have similar insensitivities, but anti-CCP is more specific and can be an indication of a more severe rheumatoid arthritis to manifest. ${ }^{13}$ Laboratory findings are also quantified according to the 2010 Rheumatoid Arthritis Classification Criteria (Table 1.) and added to the RA score.

In a RA patient the synovial fluid in the joints will have reduced viscosity without stringing, Little to no tail for each drop, and a decreased hyaluronate due to inflammation. In some cases, septic synovial fluid can manifest within the joints which will cause fluid to be opaque and purulent, with a low viscosity. Laboratory studies will show over $50.000 / \mu \mathrm{L}$ WBC count coupled with the dominant presence of polymorphonuclear leukocytes, which are usually over 75\%. Biopsies of affected joints commonly show mild proliferation around the synovial lining cells with others showing mononuclear cell infiltration and capillary cell congestion. However, the histological hallmark signs of RA are pannus invasion and articular cartilage with focal bone erosions. The only difference between early and advanced RA that can be identified 
histologically is maximal synovial lining thickness and angioneogenesis, which can occur from either mononuclear cell infiltration or synovial lining cell proliferation-indicating that angioneogenesis is one of the first signs of RA..$^{20,21}$

The before mentioned musculoskeletal symptoms of RA are the earliest manifestation of the disease. These are non-osseous and are better seen through ultrasound and MRI. These are highly sensitive radiographic imaging techniques for RA with MRI being the standard to detect early, subtle deformities. It can best detect synovial hyperplasia, rice bodies (multiple small loose bodies that resemble polished grains of rice), pannus formation, subchondral cysts, and cartilage erosion. Contrast enhancement distinguishes pre-erosions and erosions themselves from subchondral cysts that prove degenerative. Ultrasounds tend to visualize synovial proliferation and inflammation. Despite these methods radiographs and CT imaging are still used for diagnosing RA as well. ${ }^{22}$ Radiographical images of verifiable erosions occur in $30 \%$ of all patients upon diagnosis, with up to $70 \%$ within a period of 3 years.

Articular radiographic imaging in RA patients show soft tissue swelling which can either be fusiform or periarticular. Focal bone erosions exhibit juxta-articular for early-stage RA and generalized in advance. The most important use of radiographic imaging for RA, however, is to determine the extent of joint space narrowing, which can be symmetrical or concentric, as these determine the pain severity of the patient. ${ }^{23}$ Though CT is not used for articular evaluation in RA patients, it is applied for the cervical spine imaging and peri-operative assessment. ${ }^{15,24}$

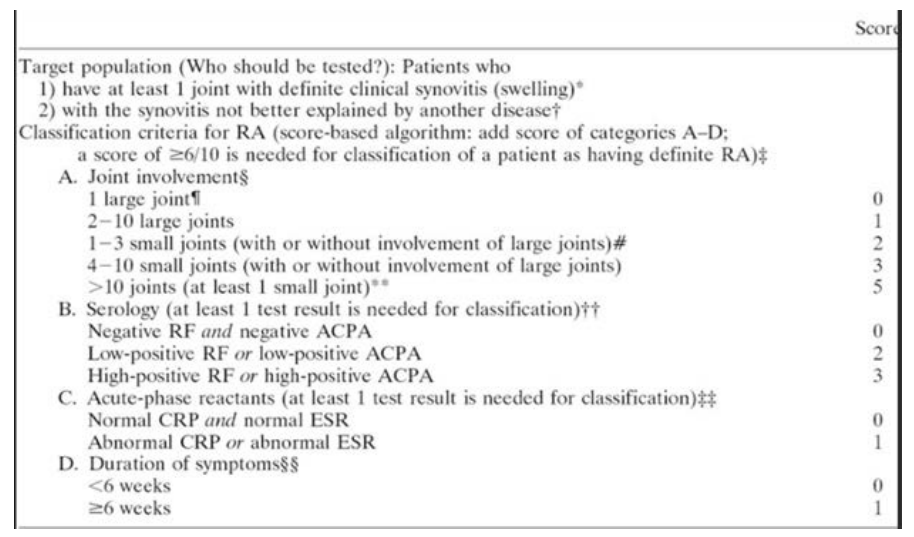

Figure 1. The American College of Rheumatology of the year 2010. ${ }^{9}$ This criterion aims at classifying patients who present with arthritic symptoms. Additionally, patients who have an erosive disease, which is common of rheumatoid arthritis, and also have a medical that fulfils the criteria from the years before 2010. This criterion classifies patients into those with RA, those suffering from a longstanding disease, those who have an inactive disease (who have undergone treatment or have no history of treatment), and those who upon analysis of present data fulfil the criteria above are classified as RA patients.

Diagnosis varies among patients who present symptoms differently. Most patients often include conditions like psoriatic arthritis, gout, and systemic lupus erythematosus. There remains debate on the appropriate diagnosis to follow; however, a rheumatologist specialist should always be available for consultation. Though patients who score 6/10 cannot be classified as rheumatoid patients it is advised that they still undergo assessment again in the future as RA progression worsens a.

$\S$ Joint involvement denotes tender or swollen joints. These joints can be assessed through imaging that proves the presence of synovitis. First carpometacarpal, first metatarsophalangeal, and Distal interphalangeal joints are all always excluded from the standard evaluation. One categorizes joint distribution and places a form of classification depending on the number of joints that happen to be affected and their location. This information is further supported by placing the associated joints into the highest category possible according to the joints involved.
When one mentions "Large joints" they are commonly referring to elbows, hips ankles, among others. "Small joints" therefore indicate locations as the proximal interphalangeal joints, thumb interphalangeal joints, metatarsophalangeal joints, and others. This category mandates that a bare minimum of one joint among the total number of involved joints should fall under the small joint classification. The remaining can be in the form of large or other small joints, and even combine with other unclassified joints such as acromioclavicular, sternoclavicular and temporomandibular among others.

Negative denotes $I U$ values which are equal to or less than the determined upper limit of normal (ULN) from assay or laboratory assessments; low-positive denotes $I U$ figures that happen to be higher than $U L N$ but $\leq 3$ times lower; A high-positive denotes $I U$ numbers that happen to be $>3$ times the ULN value. In situations where the rheumatoid factor appears only as either positive or negative, a positive outcome appears as a low RF positive. Normal/abnormal falls under local laboratory standards where CRP is the Creactive protein, and ESR is the erythrocyte sedimentation rate. The duration of symptoms denotes a patient's self-report on synovitis signs or symptoms that happen to be involved during assessment, regardless of treatment status.

\section{Indications of Surgery}

\section{Rheumatoid arthritis}

There are basically four distinct stages of rheumatoid arthritis:

Stage one: Stage one of rheumatoid arthritis is the autoimmune stage. During the stage, the body attacks the joints of the affected body parts, such as the knee, the ankles, or the wrist. Patients at the initial stage experience less severe symptoms such as stiffness in the hand, knee, or in the foot joints. ${ }^{25}$ Patients undergo different types of tests to help determine the disease considering it is not easy to conclude with a single test. Specific tests that require imaging, such as X-rays, MRI, and ultrasounds, are also considered when examining any damage to the joint. There are also signs of synovium inflammation as a result of the collection of the synovial fluid. Treatment for the condition of the synovial inflammation requires a patient to take some rest, and often if there is pain, the doctor may prescribe anti-inflammatory medications. In case the patient does not respond, the patients undergo a synovectomy after referral by an orthopedic surgeon.

During Synovectomy, surgery eliminates some of the affected tissues, or in some cases, when the patient is experiencing excessive pain, the entire tissue is removed. Some of the vulnerable joints include the knee joint, the hip joint, and the hip joint. In case the patient is not diagnosed and treated during the first stage, the synovium inflammation prolongs causes damage to the cartilage tissues. An individual requires medical diagnosis in the early stages to help determine the disease for subsequent treatment to take place.

Stage two: Lack of diagnosis and treatment in the first rheumatoid arthritis stage, to stop the synovium inflammation, damage the cartilage tissues. Usually the in the cartilage tissue, there is a connecting link between the macrophage-like and the fibroblast-like synovial cell. The cells' antibodies in the form of proteins digest the cartilage. The digestion of the cartilage cells by the proteins leads to, loss of proteoglycan that is usually present in the superficial zones. Degradative enzymes present in the polymorphonuclear white blood cells, in the synovial fluid, causes damage to the cartilage cell. ${ }^{26}$ During the second stage, a patient experiences moderate pain in the joints and reduced mobility. Patients can still undergo surgery at the second stage to help the removal of a joint and, in that case, manage pain. Removal of a joint also helps recover mobility of the affected part, such that the patient can now perform some task. Examples of such tasks include holding objects on their own if the affected part was the wrist or walking along after treating the ankle. If the patient is not treated during the second stage, the disease prolongs the third stage, which is chronic.

Severe rheumatoid arthritis: Progression of the disease from the sec- 
ond stage leads to a more severe and chronic stage, severe rheumatoid arthritis. Synovium inflammation leads to cartilage destruction, joint aches, and bones. ${ }^{27}$ Usually, there is friction between the cushion that leads to wearing out of the bones. Signs and symptoms such as bent joints, reduced mobility, excessive pain, and swelling are evident, and therefore, blood testing is not necessary. Physical deformities in some parts are also visible, and the patients may consider surgery to help manage excessive pain and, in some cases, recover lost mobility. The damage to a particular joint caused by rheumatoid arthritis occurs on either side of the body. That means that if there is damage on the joints on one of the legs, the other leg will also be affected after some time. The stage is chronic and, if not managed early enough, may lead to the death of the patient.

Progressive rheumatoid arthritis: Progressive rheumatoid arthritis is the last and fourth stage rheumatoid arthritis, where the patients no longer experience inflammation but ankylosis. Ankylosis refers to the process that causes loss of strength in the muscles. ${ }^{27}$ The stage usually is chronic, and negligence on the part of the medical doctors. During the progressive stage, the patients' joints completely malfunction, and no fusion of the muscles takes place. Patients experience excessive pain, loss of mobility, and stiffness in the affected area. Replacement surgery leads to the partial or permanent removal of the part of the joint, be it an ankle or wrist. After the replacement surgery, the patient may recover to the extent of making the affected part function, recovering mobility, and help manage pain. Delayed treatment of the disorder at the final stage can damage various body systems, such as lungs, skin blood vessels, among many more.

Indications for surgical intervention: There are various types of surgery recommended to patients who are at the chronic stages of rheumatoid arthritis. Common types of surgery include Synovectomy, Arthrodesis, and joint replacement. ${ }^{28}$ Some common signs prevalent at the chronic stage of rheumatoid arthritis include progressive deformities of the joints, malfunctioning of the affected organ, or inflamed synovial tissue. During joint replacement, the patient's bone, such as hip or knees, is replaced by either permanently or partially removing the damaged part of the joint. The resultant effects of the surgery to the patients include a reduction in pain on the affected area and recovery of mobility Arthrodesis entails the removal of the damaged joint while fusing the bones. Common joints that undergo Arthrodesis include the toes, finger joints, and wrist or ankle joints. Synovectomy, on the other hand, refers to synovial tissue removal around the joint. A synovectomy procedure helps remove the synovium tissue that results in reduced pain and other symptoms such as stiffness at the early stages of rheumatoid arthritis. Synovectomy, when done at an early stage, saves the patient from experiencing damage to the cartilage tissues. It also saves the patient from experiencing more severe pain that is a result of damage to the bones and joints. Table 1 summarizes the surgical interventions that were mentioned above.

\section{Techniques}

As with most malignancies, the clinical progression of RA will determine the treatment an individual with the severity of joint inflammation as the driving factor being that it is "most important cause of disability in the early stages". ${ }^{29}$ For many the first step taken is pharmacological with emphasis on early intervention.

Disease-modifying antirheumatic drugs (DMARDs) are the primary drug of choice for rheumatic disease. Traditional, or synthetic, DMARDS work by suppressing the immune system and is noted to have a slow response rate before a significant decrease in pain and inflammation is felt. Methotrexate is a popular synthetic due to its therapeutic properties as it is "generally well tolerated and often produces a beneficial effect in 2-6 weeks" with the usual initial dose of "7.5 or $10 \mathrm{mg}$ orally once weekly"; $; 0$ dosage can be increased if tolerance is met. It works by "stimulat[ing] adenosine release from fibroblasts, reduc[ing] neutrophil adhesion, inhibit[ing] leukotriene B4 synthesis, local IL-1, IL-6, and IL-8 production, and synovial collagenase gene expression with overall suppression of cell mediated immunity. If regimen is begun early on it is noted to prevent irreparable joint damage and deformity from rheumatic disease. Biological DMARDS are newer and genetically engineered to inhibit of tumor necrosis factor (TNF) through IV infusions. Results are expected to be available within a few weeks and thus are preferred for those who have not responded well to Methotrexate. Currently Biological DMARDS are increasingly used as initial therapy in combination with Methotrexate for patients with poor prognostic factors.

Low-dose glucocorticoids, as with oral Prednisone, produces an anti-inflammatory effect which can slow the rate of articular erosion when 5-10 mg is taken daily. Low-dose therapy has been shown in prospective studies to "retard radiographic progression of joint disease" 29 however the risks outweigh the benefits as prolonged use can predispose the patient to osteoporosis or Cushing Syndrome, a condition marked by elevated levels of cortisol in the body that can leads to fluid retention and weight gain. ${ }^{16}$ Therefore, these often are used as a "bridge to reduce disease activity until the slower acting DMARDs take effect" or as adjunctive therapy for "active disease that persists despite treatment with DMARDs" ${ }^{30}$

NSAIDs were formerly viewed as the core therapy for rheumatic disease but are now considered to be adjunctive treatment for symptom management. The anti-inflammatory properties are "derived from their ability to nonselectively inhibit cyclooxygenase (COX)-1 and COX-2" and reduce the production of prostaglandins thereby inhibiting inflammation and pain. ${ }^{29}$ Although the results of clinical trials suggest that NSAIDs efficient in reducing pain, patient experience suggests that some individuals may opt for DMARDs as NSAIDS ultimately do not slow down the progression of the disease but rather masks the symptoms for a short period of time.

Orthopedic surgery plays a key role in the management of joint destruction by rheumatic disease by "correcting deformities, stabilizing joints, decreasing disability" to improve quality of life. ${ }^{31-33}$ Most patients with rheumatoid arthritis (RA) do not opt surgery as non-invasive procedures have shown to be sufficient however some may elect to do so if the severity of their condition has proven to be unbearable. The requirement for surgical treatment in RA is a "surrogate marker of destruction of joints and failed medical treatment". ${ }^{11}$ For large joints, such as the knee, hip, shoulder, or elbow, the preferred option for advanced joint disease is Arthroplasty. This procedure entails removal of the affected joint or part of the joint to be reconstructed with either a metal, plastic or ceramic device known as a prosthesis that is designed to replicate the movement of a normal, healthy joint. The results generally provide a substantial improvement in the patient's ability to carry out normal activities as it restores joint function and fixes any deformity in the patient however, "it cannot slow down or stop disease progression to any other joint" ${ }^{34}$ There are two types: Minimally Invasive Joint Replacement Surgery and Total Joint Replacement. Minimally Invasive comprises of an incision of "3-4 inches" that takes care to "to 
limit the disruption of muscles, ligaments, and tendons under the skin" so the patient will have a shorter recovery time. Total Joint Replacement has a more traditional approach with open surgery, regardless of incision, necessary for placement of a prosthesis. Although arthroplasty is successful in "90 percent of cases", possible complications may still occur such as wound infection, infection around the prosthesis, blood clotting, prosthesis malfunction due to wear and tear or dislocation, and rarely nerve injury due to damage during surgery.

Arthodesis, also known as artificial ankylosis, is a procedure which fuses two ends of a joint to reduce or eliminate pain through the use of a bone graft. This is done to relieve intractable pain that cannot be managed by medication or other commonly indicated treatments so patients will most likely is undergo this operation in the later stages of RA. ${ }^{32}$ Used primarily for joints of the spine, hand, ankle, and toes, the surgeon will remove the deteriorating joint before replacing it with the bone graft and then will complete the procedure with screws, rods, and plates to secure the connected bones. As the most frequent surgery for advanced disease, Arthodesis has a "success rate of 80 to 100 percent", depending on fixation technique and preoperative diagnosis. Complications include pain/soreness, infection, bleeding, and in rare cases nerve damage and detachment of the hardware. As with arthroplasty this too does not slow down the disease progression but rather removes the hazard altogether. ${ }^{34}$

Synovectomy is a procedure which entails the removal of the synovium of the joint, most often of the knee, is done to reduce swelling and prevent further damage to the joint. ${ }^{35}$ The synovial membrane, a specialized connective tissue which lines the articular capsule of synovial joints, is the site of synovial fluid production "a substance that lubricates and nourishes the cartilage and bones inside the capsule". ${ }^{36-40}$ With RA, the membrane becomes inflamed and ultimately destroys the cartilage and bone of the joint thus rendering the individual incapacitated. This procedure is usually performed during the early stages of the disease however a caveat is all parts of the inflammation are rarely completely removed thus recurrence is a common occurrence. There are two basic forms of synovectomy: It may be performed either as an open surgical procedure or with the aid of an arthroscope, a "thin flexible fiberoptic scope which is introduced into a joint space through a small incision" ${ }^{36}$ Magnified pictures from the camera will then projected onto a monitor, guiding the surgeon throughout the procedure. Complications include infection, bleeding within the joint, and loss of motion. ${ }^{41-44}$

The American College of Rheumatology has developed an index with which to assess the activity of the disease known as the ACR Criteria. This considers a "variety of factors to create a score assessing the amount of improvement a patient's rheumatoid arthritis has made" after treatment through the following parameters (9):

- Measurement of inflammation through the laboratory values of ESR or CRP

- An assessment of progress through the patient's perspective

- An assessment of progress through the doctor's perspective

- How much the pain the patient feels in his or her joints on a daily base

- How much the patient is able to use their joints for daily activities

The initial ACR system developed was the ACR20, which measures a "twenty percent improvement in the patient's reported pain scale and swelling in designated joints" ${ }^{37}$ Eventually ACR50 and ACR70 were integrated to indicate "a fifty or seventy percent improvement in patients" with rheumatic symptoms. ${ }^{37}$ If a patient were to receive a score of ACR 20, this means the patient has "improved their tender and painful joint counts by 20 percent, as well as made a 20 percent improvement in three of the five above parameters" ${ }^{37}$

\section{CONCLUSION}

This research highlights the rheumatoid arthritis disease. This illness affects the joints and induces inflammation, swelling, and in severe cases can cause total immobility of the joint. Though most argue that it comes with age, the studies show that it happens as a result of steady degradation or destruction of the cartilage and bone tissue surrounding a joint. In light of these, there are several treatments that can help reduce the spread of the disease, thereby saving the synovial fluid and the cartilage, which are the primary components that enable joint mobility. These treatments include drugs such as DMARDs, but in some chronic severe cases, the only option left is surgery. As research improves, there are major steps in the fight against rheumatoid arthritis, and the leaps made in the surgical front also will ensure that we all have a range of motion and mobility throughout our lives.

\section{CONFLICTS OF INTEREST}

\section{None.}

\section{REFERENCES}

1. Cojocaru M, Cojocaru IM, Silosi I, et al. Extra-articular manifestations in rheumatoid arthritis. Maedica (Buchar). 2010; 5(4): 286-291.

2. Smolen, Landewé, Breedveld, et al. EULAR recommendations for the management of rheumatoid arthritis with synthetic and biological disease-modifying antirheumatic drugs. Ann Rheum Dis. 2014; 73(3): 492-509. doi: 10.1136/annrheumdis-2013-204573

3. Smolen, Aletaha, Bijlsma, et al. Treating rheumatoid arthritis to target: recommendations of an international task force. Ann Rheum Dis. 2010; 69(4): 631-637. doi: 10.1136/ard.2009.123919.

4. Turesson C, Matteson EL. Management of extra-articular disease manifestations in rheumatoid arthritis. Curr Opin Rheumatol. 2004; 16(3): 206-211. doi: 10.1097/00002281-200405000-00007.

5. Alvarez, Collado, Daura, et al. The rheumatoid arthritis-associated allele HLA-DR10 (DRB1* 1001) shares part of its repertoire with HLADR1 (DRB1*. 0101) and HLA-DR4 (DRB ${ }^{\star}$ 0401). Arthritis Rheum. 2008; 58(6): 1630-1639. doi: 10.1002/art.23503.

6. Van Zeben, Hazes, Zwinderman, et al. Clinical significance of rheumatoid factors in early rheumatoid arthritis: Results of a follow up study. Ann Rheum Dis. 1992; 51(9): 1029-1035. doi: 10.1136/ard.51.9.1029.

7. López-Mejías, Carmona, Genre, et al. Identification of a 3'-Untranslated genetic variant of RARB Associated with carotid intima-media thickness in rheumatoid arthritis: A genome-wide association study. Arthritis Rheumatol. 2019; 71(3): 351-360. doi: 10.1002/art.40734.

8. Gøtzsche PC, Johansen HK. Short-term low-dose corticosteroids vs placebo and nonsteroidal antiinflammatory drugs in rheumatoid arthritis. Cochrane database of systematic reviews. 2005. doi: 10.1002/14651858.CD000189

9. Combe, Landewe, Daien, et al. 2016 update of the EULAR recom- 
mendations for the management of early arthritis. Ann Rheum Dis. 2017; 76(6): 948-959. doi: 10.1136/annrheumdis-2016-210602.

10. Louie GH, Ward MM. Changes in the rates of joint surgery among patients with rheumatoid arthritis in California, 1983-2007. Ann Rheum Dis. 2010; 69(5): 868-871. doi: 10.1136/ard.2009.112474.

11. Horton MR. Rheumatoid arthritis associated interstitial lung disease. Critical reviews in computed tomography. 45: 429-440.

12. Generali E, Cantarini L, Selmi C. Ocular involvement in systemic autoimmune diseases. Clin Rev Allergy Immunol. 2015; 49(3): 263-270. doi: 10.1007/s12016-015-8518-3.

13. Longo, Fauci, Kasper, et al. Harrison's principles of internal medicine 18E Vol 2 EB. McGraw Hill Professional.

14. Walker BR, Colledge NR. Davidson's Principles and Practice of Medicine E-Book. Elsevier Health Sciences. 2013.

15. Suresh E. Diagnosis of early rheumatoid arthritis: what the non-specialist needs to know. J R Soc Med. 2004; 97(9): 421-424. doi: 10.1258/ jrsm.97.9.421.

16. Gillick JL, Wainwright J, Das K. (2015: Rheumatoid arthritis and the cervical spine: a review on the role of surgery. Int J Rheumatol. 2015; 2015: 252456. doi: 10.1155/2015/252456.

17. Gaffo A, Saag KG, Curtis JR. Treatment of rheumatoid arthritis. Am J Health Syst Pharm. 2006; 63(24): 2451-2465. doi: 10.2146/ajhp050514.

18. Zakrzewska, Sibiński, Kozłowski, et al. Hand deformity in adult rheumatoid arthritis and juvenile chronic arthritis. Chir Narzadow Ruchu Ortop Pol. 2009; 74(5): 283-288.

19. Aletaha, Neogi, Silman, et al. 2010 rheumatoid arthritis classification criteria: an American College of. Rheumatology/European League Against Rheumatism collaborative initiative. Ann Rheum Dis. 2010; 69(9): 1580-1588. doi: 10.1136/ard.2010.138461.

20. Blüml S, Smolen JS. Rheumatoid arthritis. In: The Autoimmune Diseases. 2019. 10.1016/B978-0-12-812102-3.00034-8

21. Baeten, Demetter, Cuvelier, et al. Comparative study of the synovial histology in rheumatoid arthritis, spondyloarthropathy, and osteoarthritis: Influence of disease duration and activity. Ann Rheum Dis. 2000; 59(12): 945-953. doi: 10.1136/ard.59.12.945.

22. Lipsky P. Abstracts presented at the Fourth International Synovitis Workshop Dallas, Texas, 21-25 April 1999.

23. Rominger, Bernreuter, Kenney, et al. MR imaging of the hands in early rheumatoid arthritis: Preliminary results. Radiographics. 1993; 13(1): 37-46. doi: 10.1148/radiographics.13.1.8426935.

24. Vives M J. Orthopedic imaging: A practical approach. J Spinal Cord Med. 2006; 29(2): 173. doi: 10.1043/1079-0268(2005)29[173:BR]2.0. $\mathrm{CO} ; 2$.

25. Sommer, Kladosek, Weiler, et al. Rheumatoid arthritis: A practical guide to state-of-the-art imaging, image interpretation, and clinical implications. Radiographics. 2005; 25(2): 381-398. doi: 10.1148/ rg.252045111.

26. Yeo, Adlard, Biehl, et al. Expression of chemokines CXCL4 and CXCL7 by synovial macrophages defines an early stage of rheumatoid arthritis. Ann Rheum Dis. 2016; 75(4): 763-771. doi: 10.1136/annrheumdis-2014-206921.
27. Catrina, Joshua, Klareskog, et al. Mechanisms involved in triggering rheumatoid arthritis. Immunol Rev. 2016; 269(1): 162-174. doi: 10.1111/imr.12379.

28. Lee, George, Khor, et al. Surgical intervention for rheumatoid arthritis and complication risks. Handbook of Systemic Autoimmune Diseases, 15: 127-160.

29. Shah A, St. Clair E. Rheumatoid Arthritis. In: Jameson J, Fauci AS, Kasper DL, Hauser SL, Longo DL, Loscalzo J. eds. Harrison's Principles of Internal Medicine, 20e New York, NY: McGraw-Hill. Website: http:// accessmedicine.mhmedical.com/content.aspx?bookid=2129\&section $\mathrm{id}=192284979$. Accessed March 18, 2020.

30. Hellmann DB, Imboden Jr. JB. Rheumatoid Arthritis. In: Papadakis MA, McPhee SJ, Rabow MW. eds. Current Medical Diagnosis and Treatment 2020 New York, NY: McGraw-Hill. Website: http://accessmedicine. $\mathrm{mhmedical} . \mathrm{com} /$ content.aspx?bookid=2683\&section id=225052524. Accessed March 20, 2020.

31. Wolfe F, Zwillich SH. The long-term outcomes of rheumatoid arthritis: A 23-year prospective, longitudinal study of total joint replacement and its predictors in 1,600 patients with rheumatoid arthritis. Arthritis Rheum1998; 41: 1072-1082. Crossref, Medline, Google Scholar

32. Wood GW III. Arthrodesis of the spine. In: Canale ST, Beaty JH, eds. Campbell's Operative Orthopaedics. Philadelphia, PA: Elsevier Mosby; 2012: chap 39.

33. Patient-reported complications after elective joint replacement surgery

34. Alazzawi S, Bardakos NV, Hadfield SG, Butt U, Beer ZH, and Field RE. The Journal of Bone and Joint Surgery. British volume 2012 94-B: $8,1120-1125$.

35. Lavorgna J, Nugent J, Sammarco J, Whiteside L. Basics of Surgery for Arthritis. University of Washington: Orthopaedics and Sports Medicine. Website: https://orthop.washington.edu/patient-care/articles/ arthritis/basics-of-surgery-for-arthritis.html. Published November 28, 2018. Accessed March 20, 2020.

36. Yoshihara H, Yoneoka D, Margalit A, Zuckerman JD. Rheumatoid arthritis patients undergoing total hip and knee arthroplasty have better in-hospital outcomes compared with non-rheumatoid arthritis patients. Clin Exp Rheumatol. 2016; 34(2): 270-275.

37. Green DW, Figgie MP, Novick N. Arthroscopic Synovectomy. Hospital for Special Surgery. Website: https://www.hss.edu/conditions_ synovectomy.asp. Published July 9, 2019. Accessed March 20, 2020

38. Rosenbaum, Lewis MD. What Is The American College Of Rheumatology (ACR) Scoring System For Rheumatoid Arthritis And How Does It Work? ABC News. ABC News Network, 14 Aug. 2008. Web. 20 Mar. 2017.

39. Complications of Joint Replacement Surgery. (n.d.). Stanford Health Care. Website: https://stanfordhealthcare.org/medical-treatments/a/arthroplasty/complications.html

40. Politi J, John H, Njus G, et al. First metatarsal-phalangeal joint arthrodesis: A biomechanical assessment of stability. Foot Ankle Int. 2003; 24(4): 332-337. doi: 10.1177/107110070302400405.

41. Redondo ML, Christian DR, Yanke AB. The role of synovium and synovial fluid in joint hemostasis. In: Yanke A, Cole B, eds. Joint Preser- 
vation of the Knee. Cham, Switzerland: Springer; 2019: 57-67.

42. Ekwall AKH, Firestein GS. Rheumatoid arthritis: Treatment. In EG Nabel et al., eds., Scientific American Medicine, chap. 1032. Hamilton, ON: BC Decker. Website: https://www.deckerip.com/decker/scientificamerican-medicine/chapter/1032/pdf. Accessed December 15, 2016.
43. Berger. Rapid Rehabilitation and Recovery with Minimally Invasive Total Hip Arthroplasty. Clin Orthop Relat Res. 2004.

44. Benjamin O, Bansal P, Goyal A, et al. Disease Modifying Anti-Rheumatic Drugs (DMARD) [Updated 2020 Jul 4]. In: StatPearls [Internet]. Treasure Island (FL): StatPearls Publishing; 2020. Website: https:// www.ncbi.nlm.nih.gov/books/NBK507863/ 